\title{
Optical polarimetry of infrared excess stars ${ }^{\star}$
}

\author{
C. Chavero ${ }^{1, \star \star, \star \star \star}$, M. Gómez ${ }^{1}$, B. A. Whitney ${ }^{2}$, and C. Saffe $e^{1, \dagger}$ \\ 1 Observatorio Astronómico de Córdoba, Laprida 854, 5000 Córdoba, Argentina \\ e-mail: [carolina; mercedes; saffe] @oac.uncor.edu \\ 2 Space Science Institute, 4750 Walnut St. Suite 205, Boulder, CO 80301, USA \\ e-mail: bwhitney@spacescience.org
}

Received 7 December 2004 / Accepted 9 March 2006

\section{ABSTRACT}

\begin{abstract}
We present $U B R V I$ polarimetry measurements for a group of 38 IRAS infrared excess stars and complement these observations with $V$-band data taken from the literature for 87 additional objects. After correcting the observed values by the interstellar contribution, we find that $48 \%$ of the analyzed sample has polarization excess. In addition, the polarization of these stars may correlate with infrared color excesses, particularly at 60 and $100 \mu \mathrm{m}$. We caution, however, that poor IRAS data quality at longer wavelengths affects this correlation. We analyze the wavelength dependence of the linear polarization of 15 polarized objects in relation to Serkowski's empirical interstellar law. We find that for 6 to 7 objects (depending on the interstellar model) the measured polarization differs significantly from the empirical interstellar law, suggesting an intrinsic origin. We analyze the polarimetry distribution of IRAS infrared excess objects in relation to the Exoplanet host stars (i.e., stars associated with at least one likely planetary mass object). The corresponding polarimetry distributions are different within a high confidence level. Finally, we compare the metallicity distributions of F and G IRAS infrared excess, Exoplanet host and field main sequence stars, and find that F-G IRAS infrared excess objects have metallicities quite similar (although not identical) to field main sequence stars and significantly different from the Exoplanet host group.
\end{abstract}

Key words. stars: circumstellar matter - stars: planetary systems - techniques: photometric - techniques: polarimetric

\section{Introduction}

Since the detection of a large infrared excess from Vega ( $\alpha$ Lyr) by the IRAS satellite (Aumann et al. 1984) a few other stars, $\beta$ Pic, Fomalhaut, and $\epsilon$ Eri (Gillett 1986), have shown similar signatures. This infrared excess is attributed to the presence of circumstellar dust $(T \sim 50-125 \mathrm{~K})$, warmed by the central object. Images have later confirmed the existence of disks associated with these stars (see, for example, Smith \& Terrile 1984; Holland et al. 1998; Greaves et al. 1998).

Apart from these four stars, about 200 objects have been identified by different authors as having infrared excesses (Backman \& Paresce 1993; Sylvester et al. 1996; Mannings \& Barlow 1998; Fajardo-Acosta et al. 1999; Sylvester \& Mannings 2000; Habing et al. 2001; Laureijs et al. 2002; Meyer et al. 2004; Gorlova et al. 2004; Beichman et al. 2005; Chen et al. 2005; Bryden et al. 2006) and/or sub-millimeter emission (Sheret et al. 2004; Carpenter et al. 2005).

The dust grains, in addition to reprocessing absorbed stellar radiation into infrared radiation, scatter and polarize the incident stellar light. The detection of intrinsic polarization levels (i.e., in

\footnotetext{
* Based on observations collected at the Complejo Astronómico El Leoncito (CASLEO) operated under agreement between the Consejo Nacional de Investigacines Científicas y Técnicas de la República Argentina and the National Universities of La Plata, Córdoba and San Juan.

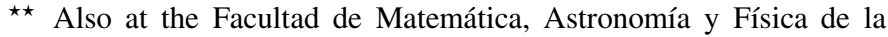
Universidad Nacional de Córdoba, Argentina.

$\star \star \star$ Present address: Observatório Nacional, Rua General José Cristino 77, São Cristovão, 20921-400 Rio de Janeiro, Brasil.

On a fellowship from CONICET, Argentina.
}

excess to the interstellar value) can provide support to the proposition that these IRAS infrared excess stars are surrounded by dusty disks, except when they are viewed face-on and the polarization vectors cancel out.

However, the detection of intrinsic polarization levels is not an easy task as the dust in the space between the observer and a given star, produces a polarization of the stellar light. The amount of this polarization basically depends on the distance to the star. In many cases the interstellar contribution can be significant in relation to the intrinsic polarization and thus it is necessary to disentangle one from the other in order to properly analyze any non-interstellar component.

In this contribution we report polarimetry data for 38 IRAS infrared excess objects in the $U, B, V, R$, and $I$ bands. We analyzed the wavelength dependence of 15 polarized objects in relation to Serkowski's empirical interstellar law that, in general terms, describes the wavelength dependence of the interstellar polarization in the optical regimen. Several authors have detected deviations from Serkowski law in the near-infrared spectral region and provide adjustments to this relation to better fit interstellar polarization over the extended $(0.3-2.5 \mu \mathrm{m})$ spectral range (Dyck \& Jones 1978; Wilking et al. 1980, 1982). We applied the original Serkowski's relation as well as a modified version to fit the data for 15 polarized objects and searched for deviations from the interstellar dependence.

We supplement our $V$-band polarimetry measurements with those of 87 additional objects obtained from the literature, and compare the polarization properties of IRAS infrared excess objects with those of Exoplanet host stars. These are stars associated with at least one likely planetary mass object, $M \sin i<$ $17 M_{\mathrm{JUP}}$, detected by high precision Doppler spectroscopy 
measurements (see, for example, Butler et al. 2003). Exoplanet host stars are, on average, metal-rich in comparison to nearby stars (Santos et al. 2004). The relatively high metallicity is a peculiarity of these objects that distinguishes them from similar stars not known to be associated with (giant) planets. We analyze the metallicity distribution of $42 \mathrm{~F}$ and $\mathrm{G}$ stars from our IRAS infrared excess object list, using data taken from the literature; and compare to the Exoplanet host stars and to main sequence stars not known to belong to either of these two groups. Our aim is to test whether the metallicity differentiates IRAS infrared excess stars from stars with Doppler detected planetary mass objects.

This paper is organized in the following manner: in Sect. 2 we introduce our sample. The observations are described in Sect. 3. In Sect. 4 we present our polarimetry analysis and results for the IRAS infrared excess stars. We compare the polarimetry and metallicity of this group of stars with those of the Exoplanet host stars in Sect. 5. We finally summarize our results in Sect. 6.

\section{The sample}

We compiled a list of 210 IRAS infrared excess stars from the literature, based on their infrared excesses and/or sub-millimeter emission (Backman \& Paresce 1993; Sylvester et al. 1996; Mannings \& Barlow 1998; Fajardo-Acosta et al. 1999; Sylvester \& Mannings 2000; Habing et al. 2001; Laureijs et al. 2002; Sheret et al. 2004). Most of these objects are class $V$ luminosity. They have spectral types B-K and roughly $50 \%$ of the sample (112 out of 210 ) is located relatively nearby (i.e., $\$ 300 \mathrm{pc}$ ). In general, IRAS infrared excess stars are out of the galactic plane (with $<10 \%$ of the sample having $|b|<10 \mathrm{deg}$ ) whereas the distribution in galactic longitude is almost random. The relative scarcity of IRAS infrared excess objects on the galactic plane reflects the IRAS bias against the detection of objects with infrared excess toward very low galactic latitudes. We eliminated objects not belonging to the main sequence or with undetermined luminosity class (13 in all). In this manner our final list (197 objects in total) comprises only class $V$ luminosity stars.

IRAS positional uncertainty ellipse is usually large, and background sources lying in the IRAS bin may, in principle, be responsible for the infrared excesses rather than the proposed stars (see, for example, Sylvester \& Mannings 2000; Lisse et al. 2002; Jayawardhana et al. 2002). In addition IRAS infrared excess stars have fluxes similar to external galaxies, in particular star-burst galaxies (Backman \& Paresce 1993; Helou 1986). Nevertheless the surface number of sufficiently bright galaxies is relatively small ( $\sim 0.004$ per square arcmin, Soifer et al. 1989; Sanders et al. 1995) and then the probability of having a galaxy lying in the background of the star is rather low.

In order to make sure that the excesses detected by IRAS really correspond to the associated stars and not to any background source and in particular to a background star we visually inspected the DSS (Digitized Sky Survey) and the 2MASS (Two Micron All Sky Survey) plates to search for contaminating objects within the IRAS uncertainty ellipse. In this manner we eliminated 70 objects from our original compilation. Our final sample has 140 main sequence stars.

We obtained photometry data from the IRAS and Hipparcos databases and constructed the diagram in Fig. 1. We use the notation $V-[12], V-[25], V-[60]$, and $V-[100]$ to indicate the magnitude differences between the $V$ filter and each of the IRAS bands. To convert the IRAS fluxes to magnitudes we applied the zero points and the color corrections as specified in Kenyon \& Hartmann (1995) and Beichman et al. (1988), respectively. The solid lines represent the location of the main sequence. The compiled sample may have color excesses at 60 and $100 \mu \mathrm{m}$, and roughly half of the objects also have excesses at 12 and $25 \mu \mathrm{m}$. These infrared excesses are usually attributed to the presence of relatively cold dust in circumstellar disks. The IRAS data quality at 60 and $100 \mu \mathrm{m}$ is worse than at 12 and $25 \mu \mathrm{m}$ (Beichman et al. 1988; Moshir et al. 1989). Thus $41 \%$ of the fluxes at $60 \mu \mathrm{m}$ and $74 \%$ at $100 \mu \mathrm{m}$ are only an upper limit, whereas at 12 and $25 \mu \mathrm{m}, 82 \%$ and $74 \%$ of the sample, respectively, have IRAS quality flags 3 and 2 (FQUAL $=3,2$ ), corresponding to high and moderate quality data and thus are real measurements. The rest of the data at these wavelengths are just upper limits to the fluxes.

IRAS positions lying more than 50 arcsec from the optical stars were not included. The median difference between the IRAS source positions and the optical coordinates is 11.5 arcsec, and only $5 \%$ of the sample (7 out of 140) has separations $\gtrsim 30$ arcsec. We usually found a perfect agreement (i.e., within 1 arcsec) between the coordinates of IRAS infrared excess objects taken from the Bright Stars Catalog and the Hipparcos positions.

\section{Observations}

The polarimetry data for 38 IRAS infrared excess objects were obtained during 11 nights in March/August 2003 and in April/September 2004 with the five channel (UBVRI) photopolarimeter of the Astronomical Observatory of Torino (Piirola 1973; Scaltriti et al. 1989) attached to the CASLEO 2.15-m telescope (San Juan, Argentina). We used a diaphragm of 15.8" and obtained $4 \times 10 \mathrm{~s}$ integrations simultaneously in the five filters. Polarimetry standards (Hsu \& Breger 1982; Schmidt et al. 1992) as well as two IRAS excess stars from Bhatt \& Manoj (2000) were observed each night to check and calibrate the data. Table 1 lists the measured values for these stars. The instrumental polarization both in modulus and in zero point for position angles turned out to be negligible within the observational error (i.e., $<0.07 \%$ in modulus and $<1 \mathrm{deg}$ in position angle). In addition no systematic differences between the data from different runs were detected.

Table 2 lists our polarimetry data as well as the specific dates when each star was observed. $V$ magnitudes were obtained from Hipparcos. The errors correspond to the standard deviation of the four measurements in each filter. Multiple observations of a single source, when available, agreed with each other within the quoted errors; we averaged these data. Figure 2 shows the $U V B R I$ polarization distributions for the measured objects. The dotted lines indicate the median of each distribution.

The linear degree of polarization is defined by the sum of squared differences. Due to this fact even for non-polarized sources, noise in the individual measurements may contribute with a positive amount to the derived polarization level, overestimating the true polarization (see, for example, Wardle \& Kronberg 1974; Simmons \& Stewart 1985). To select likely polarized objects in our observed sample we have adopted the following criterion: The measured degree of polarization in a given filter must exceed the associated uncertainty at about $3 \sigma$ level (i.e., $P / \sigma \gtrsim 3$ ). We assume a Gaussian distribution of the measurements within the corresponding error bars. In Table 3 we identify 8 likely polarized sources, 7 stars only marginally polarized, and 23 objects with no polarization excess, according to this criterion and thus consistent with non-polarized targets. For the latter objects the polarization values given in Table 2 can only be taken as upper limits to the true polarization level. 

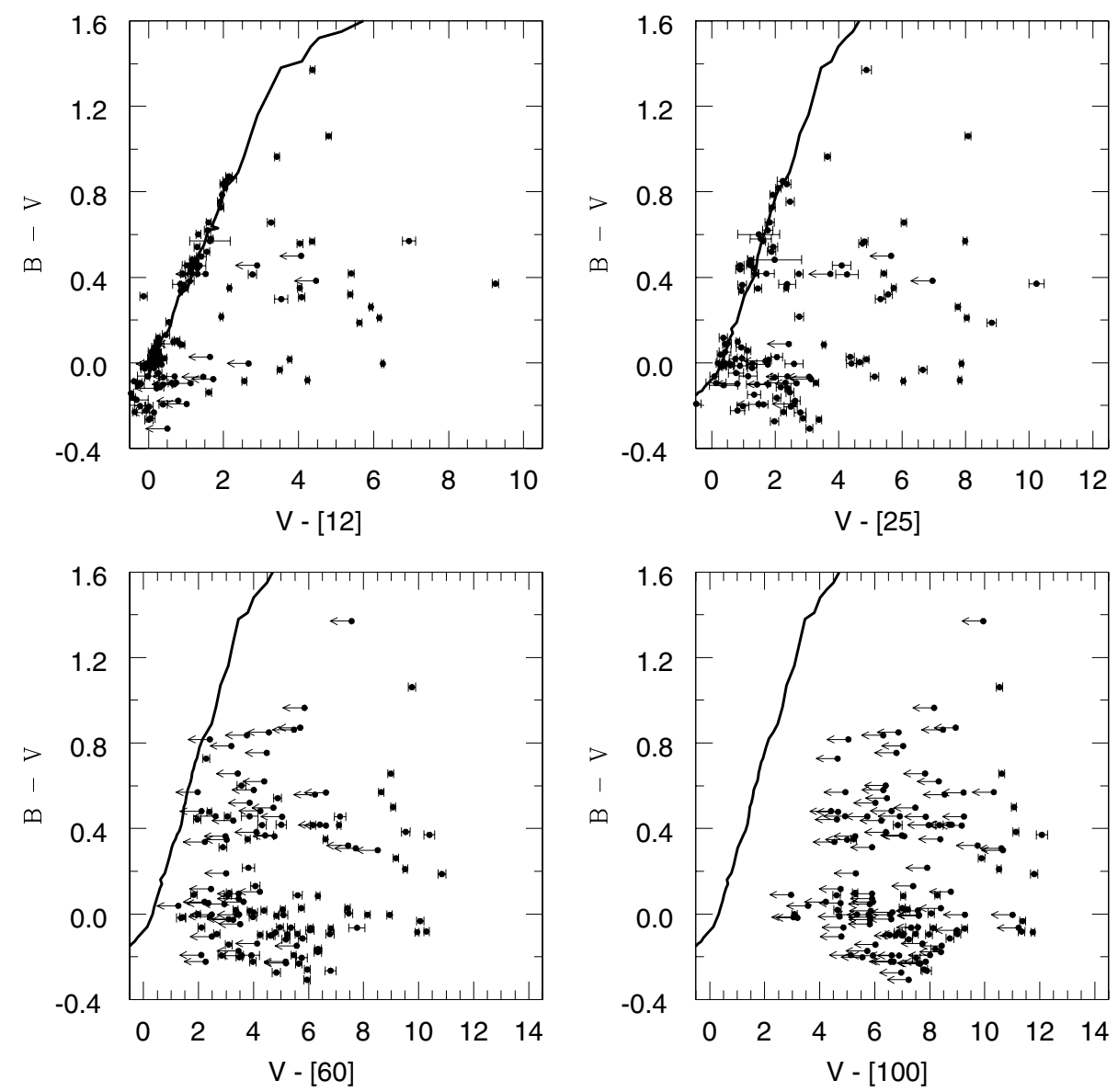

Fig. 1. $B-V$ vs. $V-$ [12], $V-$ [25], $V-[60]$, and $V-[100]$ color-color diagrams for the IRAS infrared excess objects. The error bars correspond to the uncertainties in the IRAS fluxes. The arrows indicate objects for which only upper limits to the fluxes are given by the IRAS catalog. The solid line in the upper left panel indicates the location of main sequence stars (Kenyon \& Hartmann 1995). In the other panels we use a black body to calculate the main sequence. In these cases we adopt the calibration $T_{\text {eff }}$ vs. $B-V$ from the same authors.

Table 1. Standard stars.

\begin{tabular}{|c|c|c|c|c|c|c|c|c|c|c|}
\hline Name & $\overline{P P_{U}[\%]}$ & $\overline{P_{B}[\%]}$ & $\bar{P} P_{V}[\%]$ & $\overline{P P_{R}[\%]}$ & $\overline{P_{I}[\%]}$ & $\overline{\theta_{U}\left[{ }^{\circ}\right]}$ & $\overline{\theta_{B}\left[^{\circ}\right]}$ & $\overline{\theta_{V}\left[^{\circ}\right]}$ & $\overline{\overline{\theta_{R}\left[^{\circ}\right]}}$ & $\overline{\theta_{I}\left[{ }^{\circ}\right]}$ \\
\hline HD 147084 & $2.73 \pm 0.07$ & $3.52 \pm 0.04$ & $4.19 \pm 0.03$ & $4.42 \pm 0.03$ & $4.11 \pm 0.06$ & $31.7 \pm 0.9$ & $32.8 \pm 0.8$ & $32.4 \pm 0.9$ & $31.8 \pm 0.8$ & $32.0 \pm 0.8$ \\
\hline HD 155528 & $4.22 \pm 0.06$ & $4.65 \pm 0.07$ & $4.94 \pm 0.06$ & $4.75 \pm 0.05$ & $4.18 \pm 0.06$ & $91.8 \pm 0.4$ & $92.3 \pm 0.4$ & $92.5 \pm 0.3$ & $92.4 \pm 0.5$ & $91.9 \pm 0.6$ \\
\hline HD 43955 & $0.19 \pm 0.05$ & $0.11 \pm 0.05$ & $0.09 \pm 0.04$ & $0.10 \pm 0.05$ & $0.23 \pm 0.07$ & & & & & \\
\hline HD 80558 & $2.27 \pm 0.06$ & $2.96 \pm 0.05$ & $3.10 \pm 0.04$ & $3.29 \pm 0.05$ & $2.79 \pm 0.07$ & & & & & \\
\hline HD 153968 & $0.60 \pm 0.07$ & $0.88 \pm 0.03$ & $0.91 \pm 0.07$ & $0.90 \pm 0.06$ & $1.07 \pm 0.06$ & & & & & \\
\hline
\end{tabular}

Since most of the objects have low polarization values, the position angles have large uncertainties. Table 4 gives this quantity for 10 of the 38 observed stars. For the rest of the stars $(28$ in total), relatively large uncertainties preclude us from measuring the position angles.

\section{Results}

\subsection{V-band polarimetry analysis}

\subsubsection{Polarization distribution}

To supplement our observations, we compiled $V$-band polarization data for 29 IRAS infrared excess objects from Bhatt \& Manoj (2000). Additional data for this group of objects were obtained from the Oudmaijer et al. (2001) and the Heiles (2000) catalogs. Combining our observations with the data from the literature we obtained a list of 125 objects along with their corresponding uncertainties. This compilation includes the 29 IRAS infrared excess stars measured by Bhatt \& Manoj (2000). As stated previously, most of the IRAS infrared excess objects have distances $\lesssim 300 \mathrm{pc}$ and are located off the galactic plane. The analyzed sample contains 89 IRAS infrared excess stars within this distance and 18 objects with no distance determinations.

In the following analysis we have only included stars with distances $\lesssim 300 \mathrm{pc}$. The polarization measurements corresponding to objects with $P / \sigma \lesssim 3$ represent only upper limits to the true polarization levels. In total 37 stars (i.e., $52 \%$ of the sample or 37 out of 71 objects) are consistent with unpolarized sources for which we have assumed $P=0 \%$. We have used the distances and the galactic latitudes of these objects to correct the observed polarization in the $V$-band by the interstellar contribution.

For objects within $50 \mathrm{pc}$ no correction was necessary as the interstellar contribution is negligible (Piirola 1977; Perry et al. 1982; Korhonen \& Reiz 1986; Leroy 1993). This comprises 53\% (47 out of 89 ) of the sample. Two IRAS infrared excess stars are located at high galactic latitude $(|b| \gtrsim 60 \mathrm{deg})$ and have dis- 
Table 2. UBVRI polarimetry data for IRAS infrared excess stars.

\begin{tabular}{|c|c|c|c|c|c|c|c|}
\hline Name & $\bar{V}$ & $\overline{P_{U}[\%]}$ & $\overline{P_{B}[\%]}$ & $\overline{P_{V}[\%]}$ & $\overline{\bar{P} P_{R}[\%]}$ & $\overline{P_{I}[\%]}$ & Date \\
\hline HD 3003 & 4.96 & $0.13 \pm 0.04$ & $0.08 \pm 0.06$ & $0.10 \pm 0.06$ & $0.10 \pm 0.06$ & $0.14 \pm 0.09$ & 2004 Sep. 15, 2004 Sep. 16 \\
\hline HD 9672 & 5.47 & $0.24 \pm 0.12$ & $0.13 \pm 0.08$ & $0.11 \pm 0.04$ & $0.12 \pm 0.05$ & $0.21 \pm 0.11$ & 2004 Sep. 16, 2004 Sep. 17 \\
\hline HD 10647 & 5.54 & $0.35 \pm 0.25$ & $0.15 \pm 0.08$ & $0.15 \pm 0.08$ & $0.10 \pm 0.04$ & $0.24 \pm 0.08$ & 2004 Sep. 16 \\
\hline HD 10700 & 3.45 & $0.30 \pm 0.20$ & $0.18 \pm 0.15$ & $0.13 \pm 0.06$ & $0.11 \pm 0.09$ & $0.15 \pm 0.11$ & 2004 Sep. 14, 2004 Sep. 16 \\
\hline HD 16157 & 9.05 & $0.45 \pm 0.24$ & $0.22 \pm 0.15$ & $0.11 \pm 0.08$ & $0.13 \pm 0.08$ & $0.11 \pm 0.07$ & 2004 Sep. 14, 2004 Sep. 16, 2004 Sep. 17 \\
\hline HD 17848 & 5.12 & $0.35 \pm 0.18$ & $0.13 \pm 0.08$ & $0.17 \pm 0.10$ & $0.18 \pm 0.06$ & $0.24 \pm 0.10$ & 2004 Sep. 16, 2004 Sep. 17 \\
\hline HD 39060 & 3.58 & & $0.11 \pm 0.05$ & $0.17 \pm 0.05$ & $0.10 \pm 0.05$ & $0.10 \pm 0.04$ & 2004 Sep. 14 \\
\hline HD 43954 & 8.20 & $0.31 \pm 0.15$ & $0.30 \pm 0.08$ & $0.23 \pm 0.07$ & $0.29 \pm 0.18$ & $0.41 \pm 0.22$ & 2003 Mar. 03 \\
\hline HD 43955 & 5.35 & $0.19 \pm 0.05$ & $0.11 \pm 0.05$ & $0.10 \pm 0.04$ & $0.10 \pm 0.05$ & $0.23 \pm 0.09$ & 2003 Mar. 03 \\
\hline HD 56192 & 9.74 & $0.11 \pm 0.08$ & $0.17 \pm 0.08$ & $0.22 \pm 0.06$ & $0.22 \pm 0.05$ & $0.25 \pm 0.15$ & 2003 Mar. 03 \\
\hline HD 66591 & 4.81 & $0.15 \pm 0.08$ & $0.14 \pm 0.05$ & $0.10 \pm 0.06$ & $0.19 \pm 0.09$ & $0.36 \pm 0.24$ & 2004 Apr. 22 \\
\hline HD 80459 & 6.99 & $0.91 \pm 0.05$ & $1.22 \pm 0.06$ & $1.10 \pm 0.08$ & $1.10 \pm 0.06$ & $0.99 \pm 0.03$ & 2004 Apr. 22 \\
\hline HD 80950 & 5.86 & $0.16 \pm 0.08$ & $0.08 \pm 0.06$ & $0.07 \pm 0.05$ & $0.11 \pm 0.03$ & $0.10 \pm 0.03$ & 2004 Apr. 22 \\
\hline HD 80951 & 5.28 & $0.25 \pm 0.18$ & $0.12 \pm 0.07$ & $0.12 \pm 0.06$ & $0.14 \pm 0.02$ & $0.21 \pm 0.04$ & 2004 Apr. 22 \\
\hline HD 88955 & 3.84 & $0.49 \pm 0.15$ & $0.23 \pm 0.11$ & $0.20 \pm 0.08$ & $0.37 \pm 0.16$ & $0.50 \pm 0.26$ & 2004 Apr. 22 \\
\hline HD 98800 & 8.09 & $0.60 \pm 0.25$ & $0.31 \pm 0.14$ & $0.26 \pm 0.15$ & $0.28 \pm 0.13$ & $0.29 \pm 0.19$ & 2003 Mar. 03 \\
\hline HD 105686 & 6.16 & $0.33 \pm 0.05$ & $0.26 \pm 0.07$ & $0.24 \pm 0.09$ & $0.18 \pm 0.05$ & $0.17 \pm 0.13$ & 2004 Apr. 22 \\
\hline HD 107439 & 9.03 & $4.16 \pm 0.49$ & $3.20 \pm 0.29$ & $2.34 \pm 0.11$ & $2.29 \pm 0.07$ & $2.08 \pm 0.17$ & 2004 Apr. 22 \\
\hline HD 108257 & 4.65 & $0.16 \pm 0.05$ & $0.15 \pm 0.11$ & $0.13 \pm 0.05$ & $0.10 \pm 0.04$ & $0.17 \pm 0.06$ & 2004 Apr. 23 \\
\hline HD 109573 & 5.78 & $0.20 \pm 0.05$ & $0.11 \pm 0.07$ & $0.11 \pm 0.03$ & $0.05 \pm 0.06$ & $0.08 \pm 0.08$ & 2004 Apr. 23 \\
\hline HD 113766 & 7.56 & $0.24 \pm 0.09$ & $0.17 \pm 0.03$ & $0.20 \pm 0.06$ & $0.14 \pm 0.04$ & $0.19 \pm 0.01$ & 2004 Apr. 22 \\
\hline HD 117360 & 6.52 & $0.42 \pm 0.38$ & $0.20 \pm 0.11$ & $0.14 \pm 0.05$ & $0.20 \pm 0.07$ & $0.18 \pm 0.12$ & 2004 Apr. 22 \\
\hline HD 128760 & 8.11 & $0.26 \pm 0.25$ & $0.13 \pm 0.06$ & $0.12 \pm 0.05$ & $0.10 \pm 0.06$ & $0.28 \pm 0.07$ & 2004 Apr. 23 \\
\hline HD 145263 & 8.48 & $0.63 \pm 0.13$ & $0.65 \pm 0.08$ & $0.66 \pm 0.11$ & $0.67 \pm 0.07$ & $0.57 \pm 0.17$ & 2003 Aug. 27 \\
\hline HD 150193 & 7.16 & $3.59 \pm 0.19$ & $4.79 \pm 0.51$ & $5.09 \pm 0.16$ & $5.00 \pm 0.47$ & $4.52 \pm 0.84$ & 2003 Aug. 27, 2004 Sep. 14 \\
\hline HD 150638 & 6.46 & $0.12 \pm 0.08$ & $0.22 \pm 0.09$ & $0.28 \pm 0.11$ & $0.28 \pm 0.16$ & $0.36 \pm 0.20$ & 2004 Apr. 22, 2004 Sep. 14, 2004 Sep. 15, 2004 Sep. 17 \\
\hline HD 153968 & 9.20 & $0.60 \pm 0.07$ & $0.88 \pm 0.04$ & $0.91 \pm 0.07$ & $0.92 \pm 0.05$ & $0.91 \pm 0.18$ & 2003 Aug. 29 \\
\hline HD 158643 & 4.80 & $0.50 \pm 0.07$ & $0.57 \pm 0.06$ & $0.45 \pm 0.11$ & $0.35 \pm 0.05$ & $0.38 \pm 0.12$ & 2003 Aug. 27, 2004 Sep. 14, 2004 Sep. 15 \\
\hline HD 161868 & 3.60 & $0.11 \pm 0.10$ & $0.11 \pm 0.02$ & $0.05 \pm 0.03$ & $0.09 \pm 0.05$ & $0.11 \pm 0.01$ & 2003 Aug. 28 \\
\hline HD 165088 & 9.76 & $0.25 \pm 0.12$ & $0.41 \pm 0.05$ & $0.41 \pm 0.07$ & $0.42 \pm 0.07$ & $0.45 \pm 0.12$ & 2003 Aug. 27, 2004 Sep. 14, 2004 Sep. 15 \\
\hline HD 169142 & 7.09 & $0.29 \pm 0.17$ & $0.21 \pm 0.07$ & $0.23 \pm 0.19$ & $0.21 \pm 0.15$ & $0.27 \pm 0.13$ & 2003 Aug. 27, 2004 Sep. 14 \\
\hline HD 178253 & 3.96 & $0.19 \pm 0.10$ & $0.13 \pm 0.08$ & $0.12 \pm 0.07$ & $0.10 \pm 0.08$ & $0.14 \pm 0.07$ & 2003 Aug. 29, 2004 Sep. 14, 2004 Sep. 15, 2004 Sep. 16 \\
\hline HD 181327 & 6.84 & $0.25 \pm 0.16$ & $0.12 \pm 0.04$ & $0.08 \pm 0.03$ & $0.09 \pm 0.05$ & $0.13 \pm 0.08$ & 2004 Sep. 17 \\
\hline HD 181869 & 3.96 & $0.14 \pm 0.07$ & $0.09 \pm 0.04$ & $0.06 \pm 0.03$ & $0.05 \pm 0.02$ & $0.11 \pm 0.09$ & 2004 Sep. 15 \\
\hline HD 203608 & 4.10 & $0.24 \pm 0.12$ & $0.13 \pm 0.06$ & $0.10 \pm 0.06$ & $0.08 \pm 0.04$ & $0.09 \pm 0.05$ & 2003 Aug. 29, 2004 Sep. 15, 2004 Sep. 16, 2004 Sep. 17 \\
\hline HD 207129 & 5.58 & $0.18 \pm 0.10$ & $0.15 \pm 0.05$ & $0.09 \pm 0.05$ & $0.07 \pm 0.04$ & $0.12 \pm 0.08$ & 2004 Sep. 16, 2004 Sep. 17 \\
\hline HD 216956 & 1.17 & $0.42 \pm 0.16$ & $0.22 \pm 0.13$ & $0.11 \pm 0.05$ & $0.12 \pm 0.07$ & $0.13 \pm 0.06$ & 2004 Sep. 14, 2004 Sep. 15, 2004 Sep. 17 \\
\hline HD 224392 & 4.99 & $0.30 \pm 0.15$ & $0.07 \pm 0.04$ & $0.11 \pm 0.06$ & $0.13 \pm 0.09$ & $0.18 \pm 0.13$ & 2004 Sep. 14,2004 Sep. 15,2004 Sep. 16 \\
\hline
\end{tabular}

tances greater than $50 \mathrm{pc}$. For these objects we used the mean ISM polarization versus distance determination from Berdyugin et al. (2004) to correct the observed values. Finally for the objects located at distances greater than $50 \mathrm{pc}$ and at relatively low and intermediate galactic latitude $(5 \mathrm{deg} \lesssim|b| \lesssim 60 \mathrm{deg})$ we used the interstellar extinction versus distance curves from Fresneau (1994) to derive the ISM absorption to each star by linear interpolation. These authors determine $A_{\mathrm{B}}$ for distances between 90 and 360 pc. We adopted $R=3$ and then used $A_{\mathrm{V}}=0.75 A_{\mathrm{B}}$ to obtain the extinction in the $V$-band. We applied the relation $P_{\mathrm{V}}^{\mathrm{ISM}}=A_{\mathrm{V}}^{\mathrm{ISM}}$ to correct the observed values by the mean interstellar contribution (see, for example, Serkowski 1963; Serkowski et al. 1975; Reiz \& Franco 1998; Whittet et al. 2001).

Figure 3 shows the histogram distribution for the 71 IRAS excess stars within $300 \mathrm{pc}$, after correcting for the ISM contribution. $52 \%$ of the sample ( 37 out of 71 sources) have $P \sim 0 \%$. On the other hand, $48 \%$ of the stars ( 34 out of 71 objects) have polarization excess levels over the general ISM, likely of intrinsic origin and probably due to the presence of dusty circumstellar material. Moreover fourteen these stars (i.e., $20 \%$ of the sample) have optical polarization levels $>0.10 \%$. Two IRAS excess stars with polarization $>1$ lie outside the range plotted in Fig. 3 . Unfortunately we can not discard non-Gaussian errors as the cause of these outliers. Following-up observations of these stars are necessary to confirm these measurements.

\subsubsection{V-band polarization vs. infrared color excesses}

Intrinsic visible polarization levels and infrared color excesses are attributed to the same physical cause, i.e., the presence of dust in circumstellar disks. Figure 4 shows the polarization values of IRAS infrared excess objects vs. the color excesses at 12, 25,60 , and $100 \mu \mathrm{m}$. A positive correlation between the $V$-band polarization and the color excesses is apparent at 60 and $100 \mu \mathrm{m}$. On the contrary, no clear tendency is seen at 12 and $25 \mu \mathrm{m}$. Unfortunately, as mentioned before, poor IRAS data quality affects fluxes at 60 and $100 \mu \mathrm{m}$. This fact casts some doubts on the reality of the detected correlation. In addition for about $38 \%$ of the sample (38 out of 101 sources) only upper limits to the polarization values are available. Most of these targets have very low polarizations (i.e., $P \lessgtr 0.05 \%$ ).

Bhatt \& Manoj (2000) also found a positive correlation between the polarization and the $L_{\mathrm{IR}} / L_{*}$, the excess infrared luminosity (derived from IRAS data) over the bolometric stellar luminosity, for the sample they analyzed. Better quality data (i.e., for example, Spitzer Space Telescope observations) can provide more reliability to the correlation between the optical 

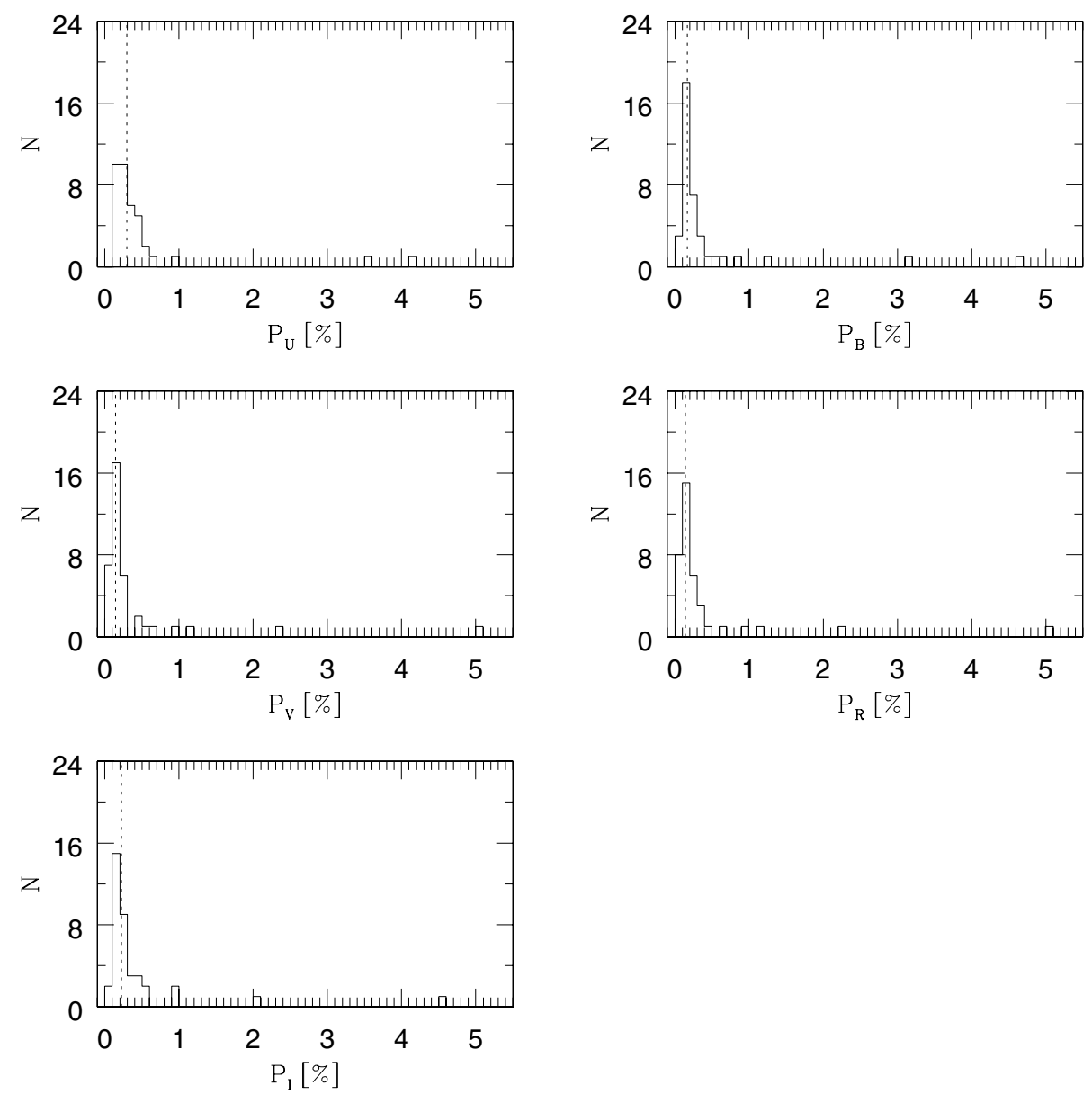

Fig. 2. $U B V R I$ polarization distributions for the observed stars. The dotted lines indicate the median of each histogram. These are: 0.24 ( $U$ band), 0.145 ( $B$ band), 0.13 ( $V$ band), 0.12 ( $R$ band), 0.175 ( $I$ band).

polarization of IRAS infrared excess stars and their infrared color excesses.

\subsection{Deviation from Serkowski interstellar law}

Serkowski et al. (1975) found that the wavelength dependence of the interstellar linear polarization over the optical range can be described by the empirical formula:

$$
p_{\lambda}=p_{\max } \exp \left[-K \ln ^{2}\left(\lambda_{\max } / \lambda\right)\right]
$$

where $p_{\lambda}$ is the percent of polarization at wavelength $\lambda$ and $p_{\max }$ is the peak polarization, occurring at wavelength $\lambda_{\max }$. The parameter $K$ was originally treated as a constant with value 1.15 by Serkowski et al. (1975). However, more recently, several authors have considered $K$ as a free parameter (see, for example, Wilking et al. 1982; Whittet et al. 1992). A weighted non-linear least-squares fit of Equation 1 allows us to derive the three unknown quantities: $K, p_{\max }$, and $\lambda_{\max }$.

The parameters in Serkowski's law $\left(p_{\lambda}, K\right.$, and $\left.\lambda_{\max }\right)$ are related to properties of the interstellar medium dust particles. Roughly speaking, $\lambda_{\max }$ traces the dominant size of the polarizing dust grains, $p_{\lambda}$ is related to the column density and degree of alignment of the interstellar grains, and $K$ indicates the width of the grain-size distribution.

For the stars in Table 3 identified as likely or marginally polarized (15 objects in total), we modeled the $P(\lambda)$ data using Serkowski empirical law with three free parameters $\left(p_{\lambda}, K\right.$, and $\left.\lambda_{\max }\right)$. To find the best fit, we searched the parameter space letting $\mathrm{K}$ vary between 0 and 2 and $\lambda_{\max }$ between 3000 and $9000 \AA$. We also applied the two parameter models corresponding to the original Serkowski relation (i.e., with $K=1.15$ ) and explored fits with $\lambda_{\max }$ within the same range. Table 5 shows the results of the fits. Figure 5 shows these fits for 15 analyzed stars. The continuous line corresponds to the three parameter model and the dotted line to the two parameter fit.

We find that fits to Serkowski relation which result in reduced $\chi^{2}$ 's $<1.5$ and $<2.0$ for the three- and two-parameter models, respectively, agree well with an interstellar origin for polarization. According to this criterion, 7 or 6 objects (depending on the model fit) in Table 5 show a wavelength dependence on the polarization that can not be of interstellar origin and likely include an intrinsic component to the observed polarization.

Oudmaijer et al. (2001) carried out a similar three-parameter fit for a group of $\sim 15$ IRAS infrared excess stars for which they obtained $U B V R I$ polarimetry and found that for at least four objects ( $51 \mathrm{Oph} / \mathrm{HD} 158643$, BD $+31^{\circ} 643 \mathrm{C}$, HD 58647, and HD 233517), the wavelength dependence of the polarization can not be attributed to normal interstellar dust, given by Serkowski relation. We have one object in common with the 15 stars studied by Oudmaijer et al. (2001): $51 \mathrm{Oph} / \mathrm{HD} 158643$. Oudmaijer et al. (2001) found that $51 \mathrm{Oph} / \mathrm{HD} 158643$ does not satisfy the Serkowski's law. Our analysis finds the same result.

Deviations from Serkowski relation may suggest the presence of an intrinsic polarization component (that can not be ascribed to the interstellar medium) or may simply indicate that 
Table 3. Likely, marginally and non-polarized IRAS infrared excess stars in our sample.

\begin{tabular}{|c|c|c|c|c|c|c|}
\hline Name & $P_{U} / \sigma_{U}$ & $\bar{P} P_{B} / \sigma_{B}$ & $P_{V} / \sigma_{V}$ & $\bar{P} P_{R} / \sigma_{R}$ & $\bar{P} P_{I} / \sigma_{I}$ & Description \\
\hline HD 3003 & 3.25 & 1.3 & 1.7 & 1.7 & 1.6 & unpolarized \\
\hline HD 9672 & 2.0 & 1.6 & 2.75 & 2.4 & 1.9 & marginally polarized \\
\hline HD 10647 & 1.4 & 1.9 & 1.9 & 2.5 & 3.0 & unpolarized \\
\hline HD 10700 & 1.5 & 1.2 & 2.2 & 1.2 & 1.4 & unpolarized \\
\hline HD 16157 & 1.9 & 1.5 & 1.4 & 1.6 & 1.6 & unpolarized \\
\hline HD 17848 & 1.9 & 1.6 & 1.7 & 3.0 & 2.4 & unpolarized \\
\hline HD 39060 & & 2.2 & 3.4 & 2.0 & 2.5 & marginally polarized \\
\hline HD 43954 & 2.1 & 3.75 & 3.3 & 1.6 & 1.9 & marginally polarized \\
\hline HD 43955 & 3.8 & 2.2 & 2.5 & 2.0 & 2.6 & unpolarized \\
\hline HD 56192 & 1.4 & 2.1 & 3.7 & 4.4 & 1.7 & marginally polarizec \\
\hline HD 66591 & 1.9 & 2.8 & 1.7 & 2.1 & 1.5 & unpolarized \\
\hline HD 80459 & 18.2 & 20.3 & 13.75 & 18.3 & 33.0 & polarized \\
\hline HD 80950 & 2.0 & 1.3 & 1.4 & 3.7 & 3.3 & unpolarized \\
\hline HD 80951 & 1.4 & 1.7 & 2.0 & 7.0 & 5.25 & unpolarized \\
\hline HD 88955 & 3.3 & 2.1 & 2.5 & 2.3 & 1.9 & unpolarized \\
\hline HD 98800 & 2.4 & 2.2 & 1.7 & 2.15 & 1.5 & unpolarized \\
\hline HD 105686 & 6.6 & 3.7 & 2.7 & 3.6 & 1.3 & marginally polarized \\
\hline HD 107439 & 8.5 & 11.0 & 21.3 & 32.7 & 12.2 & polarized \\
\hline HD 108257 & 3.2 & 1.4 & 2.6 & 2.5 & 2.8 & unpolarized \\
\hline HD 109573 & 4.0 & 1.6 & 3.7 & 0.8 & 1.0 & marginally polarized \\
\hline HD 113766 & 2.7 & 5.7 & 3.3 & 3.5 & 19.0 & polarized \\
\hline HD 117360 & 1.1 & 1.8 & 2.8 & 2.9 & 1.5 & marginally polarized \\
\hline HD 128760 & 1.0 & 2.2 & 2.4 & 1.7 & 4.0 & unpolarized \\
\hline HD 145263 & 4.8 & 8.1 & 6.0 & 9.6 & 3.35 & polarized \\
\hline HD 150193 & 18.9 & 9.4 & 31.8 & 10.6 & 5.4 & polarized \\
\hline HD 150638 & 1.5 & 2.4 & 2.5 & 1.75 & 1.8 & unpolarized \\
\hline HD 153968 & 8.6 & 22.0 & 13.0 & 18.4 & 5.1 & polarized \\
\hline HD 158643 & 7.1 & 9.5 & 4.1 & 7.0 & 3.2 & polarized \\
\hline HD 161868 & 1.1 & 5.5 & 1.7 & 1.8 & 11.0 & unpolarized \\
\hline HD 165088 & 2.1 & 8.2 & 5.9 & 6.0 & 3.75 & polarized \\
\hline HD 169142 & 1.7 & 3.0 & 1.2 & 1.4 & 2.1 & unpolarized \\
\hline HD 178253 & 1.9 & 1.6 & 1.7 & 1.25 & 2.1 & unpolarized \\
\hline HD 181327 & 1.6 & 3.0 & 2.7 & 1.8 & 1.6 & marginally polarized \\
\hline HD 181869 & 2.0 & 2.25 & 2.0 & 2.5 & 1.2 & unpolarized \\
\hline HD 203608 & 2.0 & 2.2 & 1.7 & 2.0 & 1.8 & unpolarized \\
\hline HD 207129 & 1.8 & 3.0 & 1.8 & 1.75 & 1.5 & unpolarized \\
\hline HD 216956 & 2.6 & 1.7 & 2.2 & 1.7 & 2.2 & unpolarized \\
\hline HD 224392 & 2.0 & 1.75 & 1.8 & 1.4 & 1.4 & unpolarized \\
\hline
\end{tabular}

Table 4. UBVRI position angles for IRAS infrared excess stars.

\begin{tabular}{lccccc}
\hline \hline Name & $\theta_{U}\left[^{\circ}\right]$ & $\theta_{B}\left[^{\circ}\right]$ & $\theta_{V}\left[^{\circ}\right]$ & $\theta_{R}\left[^{\circ}\right]$ & $\theta_{I}\left[^{\circ}\right]$ \\
\hline HD 43954 & $64 \pm 10$ & $59 \pm 16$ & $49 \pm 29$ & $62 \pm 34$ & $64 \pm 36$ \\
HD 56192 & $164 \pm 12$ & $154 \pm 16$ & $153 \pm 7$ & $153 \pm 6$ & $149 \pm 16$ \\
HD 80459 & $113 \pm 3$ & $117 \pm 3$ & $111 \pm 1$ & $107 \pm 3$ & $111 \pm 4$ \\
HD 107439 & $42 \pm 4$ & $51 \pm 5$ & $51 \pm 1$ & $49 \pm 1$ & $53 \pm 2$ \\
HD 113766 & $109 \pm 16$ & $122 \pm 24$ & $115 \pm 7$ & $123 \pm 9$ & $123 \pm 18$ \\
HD 145263 & $30 \pm 6$ & $34 \pm 5$ & $27 \pm 4$ & $23 \pm 6$ & $24 \pm 6$ \\
HD 150193 & $64 \pm 1$ & $66 \pm 2$ & $60 \pm 1$ & $57 \pm 1$ & $57 \pm 2$ \\
HD 153968 & $28 \pm 8$ & $34 \pm 4$ & $30 \pm 4$ & $26 \pm 7$ & $26 \pm 8$ \\
HD 158643 & $40 \pm 4$ & $38 \pm 3$ & $34 \pm 3$ & $30 \pm 4$ & $31 \pm 7$ \\
HD 165088 & $160 \pm 14$ & $170 \pm 12$ & $167 \pm 5$ & $148 \pm 46$ & $163 \pm 7$ \\
\hline \multicolumn{7}{c}{}
\end{tabular}

Serkowski law does not provide a good enough empirical representation of the interstellar polarization in all possible directions. In fact, several authors have argued this case (Dyck \& Jones 1978; Wilking et al. 1980, 1982; Whittet et al. 1992). For the IRAS infrared excess stars relatively cold circumstellar dust is usually invoked to produce the color excesses. This circumstellar dust may also be responsible for the deviations from Serkowski law observed for some of these stars although neither another intrinsic origin nor an inadequate representation of the interstel- lar medium in the direction toward these objects by Serkowski's relation can be ruled out.

\section{Comparison with the Exoplanet host group}

\subsection{Polarimetry analysis}

In this section we compare polarization characteristics of the Exoplanet host group with those of the IRAS infrared excess stars. Exoplanet host (EH) stars have companions with typical masses of $M \sin i<17 M_{\text {Jup }}$, detected by Doppler spectroscopy (see, for example, Butler et al. 2003). We obtained the list of stars associated with planets from the California and Carnegie Planet Search ${ }^{1}$ and the Geneva Observatory Planet Search ${ }^{2}$ surveys. The EH group reflects the observational focus on nearsolar-type stars where $\mathrm{G}$ dwarfs contribute to the majority of the detections. This is so because $\mathrm{G}$ spectral types contain many metallic atomic lines appropriate for high precision radial velocity measurements. On the contrary, most of IRAS infrared excess objects have spectral types A-F. These relatively early spectral type objects are more luminous than later spectral types thus more able to heat up circumstellar dust.

\footnotetext{
1 http://exoplanets.org

2 http://obswww.unige.ch/exoplanets
} 


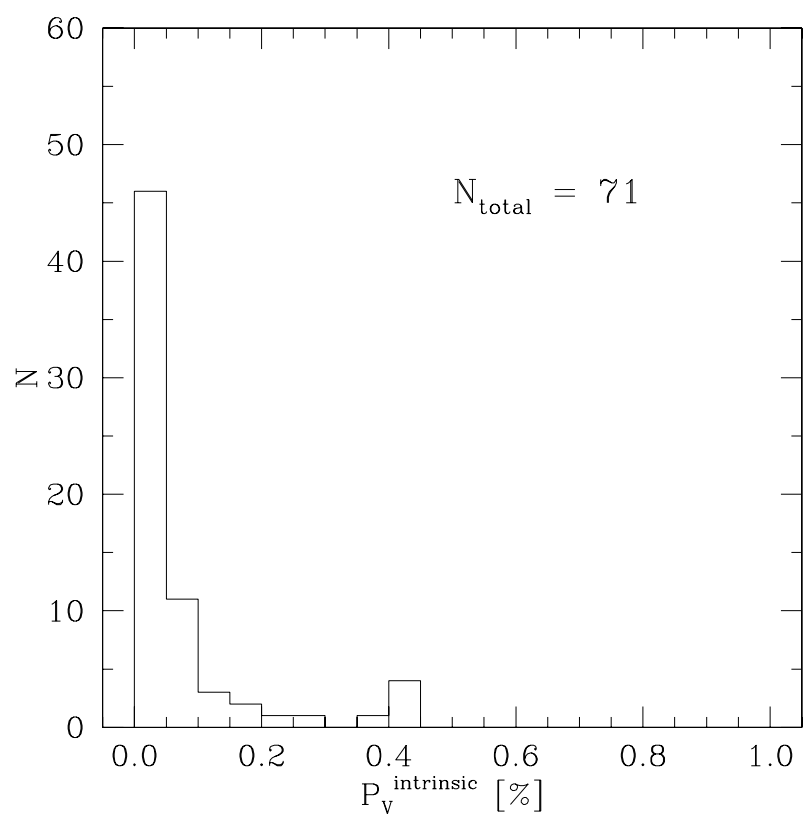

Fig. 3. Polarization excess over the mean ISM contribution for IRAS infrared excess objects. Two IRAS excess stars with polarization $>1 \%$ lie outside the range plotted in Fig. 3.

In spite of the bias or selection effects of both samples, it is interesting to compare the polarimetry properties of Exoplanet host group, with planetary mass companions, and of IRAS infrared excess stars, associated with dusty circumstellar disks which potentially form planets.

We used the Leroy (1993) and the Heiles (2000) catalogs to compile optical polarimetry data for 28 of the EH stars (see also Tamburini et al. 2002). The range of distances of these stars goes from 3 to $42 \mathrm{pc}$. The median distance of the group is $18 \mathrm{pc}$. We selected IRAS infrared excess stars within a similar range of distance (i.e., $<40 \mathrm{pc}$ ). This includes a total of 41 objects. In Fig. 6, we show these distributions. Most of the EH stars have very low polarization values, comparable to their uncertainties, except in the case of Tau Boo $(P=0.056 \pm 0.017 \%$, Heiles 2000). Basically, EH stars are non-polarized sources. The polarization measurements of 24 of the IRAS infrared excess stars with distances $<40$ pc (i.e., $58 \%$ of the sample or 24 out of 41 sources) are consistent with unpolarized targets, for which $P / \sigma<3$. However, 17 of these stars have polarization values $>0.02 \%$ (with $P / \sigma \gtrsim 3$ ) and nine objects have $P>0.05 \%$ (also at the $3 \sigma$ significance level). The KS test indicates a probability of about $0.011 \%$ for the IRAS infrared excess and the Exoplanet host polarization distributions are identical. Thus these distributions are different within a relatively high level of confidence (see also Judin 2000).

We notice that these distributions are not corrected by the effect of the interstellar contribution. However the polarization of EH stars is basically zero and thus the interstellar contribution is negligible. In the case of the IRAS infrared excess stars we have only considered stars within the same range of distance as the Exoplanet host stars. On average the interstellar contribution (if any) should be the same for both groups, even though, the group of IRAS infrared excess stars has a significant fraction of polarized objects (i.e., $41 \%$ of the sample, 17 out of 41 sources).

Figure 7 shows the positions of the Exoplanet host stars in the $B-V$ vs. $V-[12], V-[25], V-[60]$, and $V-$ [100] colorcolor diagrams. On average, the Exoplanet host group has more modest color excesses than the IRAS infrared excess sample (see
Fig. 1). The differences in color excesses as well as in polarization levels between the two groups are probably due to the existence of relatively less dust associated with the Exoplanet host stars than with the IRAS infrared excess objects.

\subsection{Metallicity analysis}

It is well established that the Exoplanet host sample is, on average, metal-rich compared to solar neighborhood field stars not known to have planets, detectable by means of high precision radial velocity measurements (González 1997; Laughlin \& Adams 1997; González 1998; González et al. 2001; Santos et al. 2001, 2004). In this section we compare the metallicities of the IRAS infrared excess stars with those of the Exoplanet host stars and main sequence stars not known to belong to either of these groups.

We obtained metallicity for $F$ and $G$ stars in the analyzed sample of IRAS infrared excess stars from the catalog of Nordström et al. (2004). These authors used Strömgren uvby photometry and the calibration of Schuster \& Nissen (1989) in their metal abundance derivations. They also improved the latter calibration by introducing a correction for the reddest stars, based on a systematic difference with high-resolution spectroscopic metallicities, and extending the original Schuster \& Nissen (1989) metallicities / Strömgren photometry relation to F stars, with relatively high temperatures.

In our analysis we adopted the metallicities of Nordström et al. (2004) when available, used their calibration to derive the metal abundances for a few objects not listed in their catalog and directly applied the calibration of Schuster \& Nissen (1989) and the Strömgren $u v b y$ photometry from the catalog of Hauck \& Mermilliod (1998) to include a few other IRAS infrared excess stars with photometry indices outside (but not far off) the range of the calibration of Nordström et al. (2004). In total we obtained metallicities for 42 IRAS infrared excess objects.

Figure 8 upper panel shows the normalized metallicity distributions of IRAS infrared excess objects (continuous line) and field stars (dotted line), taken from Nordström et al. (2004). We selected single stars within $300 \mathrm{pc}$ and eliminated IRAS infrared excess objects to define our sample of $\sim 9300$ field stars. The median of the IRAS infrared excess and field star distributions are -0.12 and -0.15 , respectively. The KS statistical test indicates a probability of $39 \%$ for the two distributions being identical. For comparison, in the lower panel of Fig. 8, we reproduce the metallicity distributions of Exoplanet hosts (continuous line) and field stars (dotted line), taken from Santos et al. (2004). The medians of these distributions are +0.17 and -0.16 , respectively. Santos et al. (2004) give a probability of the order of $10^{-9}$ for the distributions deriving from the same parent population. Thus these latter distributions are different within a high confidence level. There are two main suggestions in the literature to explain the "excess" metallicity in Exoplanet host stars: 1) a primordial origin and/or 2) accretion of metal-rich bodies. In the first case a high metal content was present in the cloud of gas and dust that gave birth to the stars with planets (see, for example, Santos et al. 2001). In the second possibility the infall of rocky or metal-rich material or planetesimals onto the the central star took place increasing the photospheric metallicity (see, for example, González et al. 2001; Murray \& Chaboyer 2002).

Recently, Fischer \& Valenti (2005) analyzed 850 FGK-type stars with high precision Doppler measurements to uniformly detect planets producing semi-amplitude variation $K>30 \mathrm{~m} \mathrm{~s}^{-1}$ and orbital period shorter than 4 days. These authors concluded that the exoplanet host group does not show an accretion 

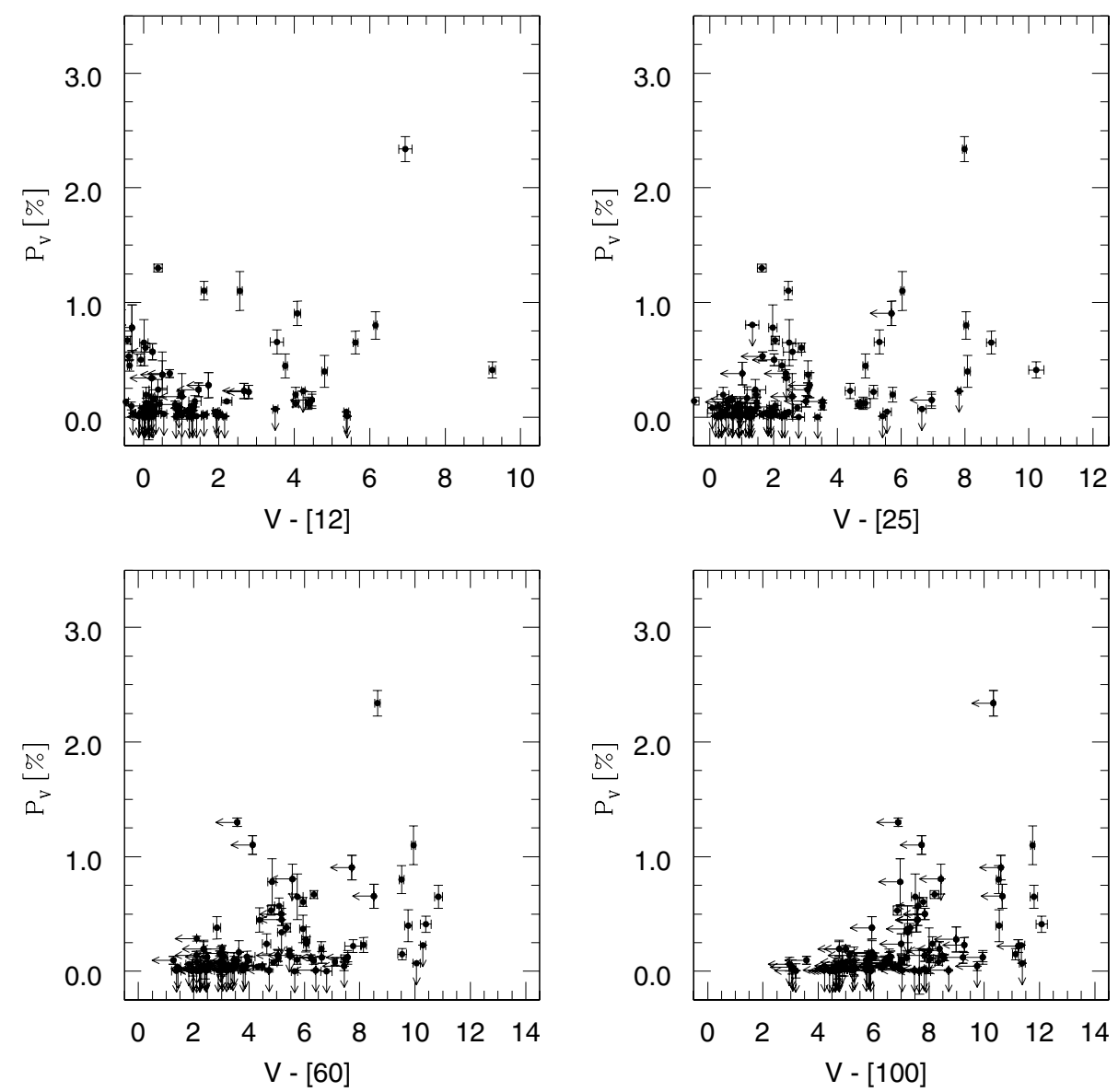

Fig. 4. Polarization of IRAS infrared excess objects vs. $V-[12]$, [25], [60], and [100] color excesses. The error bars correspond to the uncertainties in the IRAS fluxes and in the optical polarization values. The arrows indicate objects for which only upper limits to the polarization values and to the IRAS fluxes are available.

Table 5. Fits to Serkowski law.

\begin{tabular}{lccccccc}
\hline \hline Name & $\chi^{2}$ & $p_{\max }[\%]$ & $K$ & $\lambda_{\max }[\AA]$ & $\chi^{2}(K=1.15)$ & $p_{\max }[\%](K=1.15)$ & $\lambda_{\max }[\AA](K=1.15)$ \\
\hline HD 9672 & 2.00 & 0.13 & 0.02 & 3077 & 2.74 & 0.14 & 4843 \\
HD 39060 & 1.15 & 0.14 & 1.63 & 5946 & 1.16 & 0.14 & 6000 \\
HD 43954 & 0.92 & 0.28 & 0.01 & 8997 & 1.58 & 0.28 & 5091 \\
HD 56192 & 0.14 & 0.23 & 1.83 & 6609 & 0.18 & 0.23 & 7399 \\
HD 80459 & 5.11 & 1.20 & 1.23 & 5572 & 5.18 & 1.18 & 5568 \\
HD 105686 & 0.59 & 0.32 & 1.15 & 3343 & 0.59 & 0.32 & 3343 \\
HD 107439 & 14.36 & 2.86 & 0.35 & 3003 & 24.33 & 2.67 & 3687 \\
HD 109573 & 1.32 & 0.18 & 1.15 & 3024 & 1.32 & 0.18 & 6395 \\
HD 113766 & 2.13 & 0.19 & 0.04 & 8904 & 3.70 & 0.20 & 5459 \\
HD 145263 & 0.19 & 0.67 & 0.45 & 5496 & 0.49 & 0.70 & 6284 \\
HD 150193 & 0.11 & 5.20 & 1.45 & 5949 & 0.54 & 5.22 & 6036 \\
HD 153968 & 2.42 & 0.98 & 1.82 & 5772 & 3.40 & 0.96 & 3945 \\
HD 158643 & 1.64 & 0.54 & 1.34 & 4094 & 1.67 & 0.53 & 6230 \\
HD 165088 & 0.81 & 0.44 & 1.00 & 6343 & 0.82 & 0.44 & 4121 \\
HD 181327 & 1.76 & 0.10 & 0.01 & 3059 & 2.15 & 0.11 & \\
\hline
\end{tabular}

signature that might distinguish them from other stars not known to be associated with Doppler detected planes. This result suggests that the metal-rich nature of exoplanet host stars is likely due to the high metallicity content in the molecular cloud in which they were born.

IRAS infrared excess objects and field stars have similar distributions in metallicity, although not identical as indicated by the KS test. We caution, however, that no B and A spectral type IRAS infrared excess stars are included in this analysis.
On the other hand, the lack of these spectral types makes the IRAS infrared excess and EH samples homogeneous in spectral types. The KS test indicates a probability of the order of $6 \times 10^{-13}$ for the Exoplanet host and IRAS infrared excess distributions being identical and thus these groups seem to have quite different metallicities. Recently, Bryden et al. (2006) obtained Spitzer/MIPS $70 \mu \mathrm{m}$ observations for a group of 69 mainsequence field stars. They detected $70 \mu \mathrm{m}$ excess around 7 of these stars at the $3 \sigma$ confidence level. In addition, these authors 

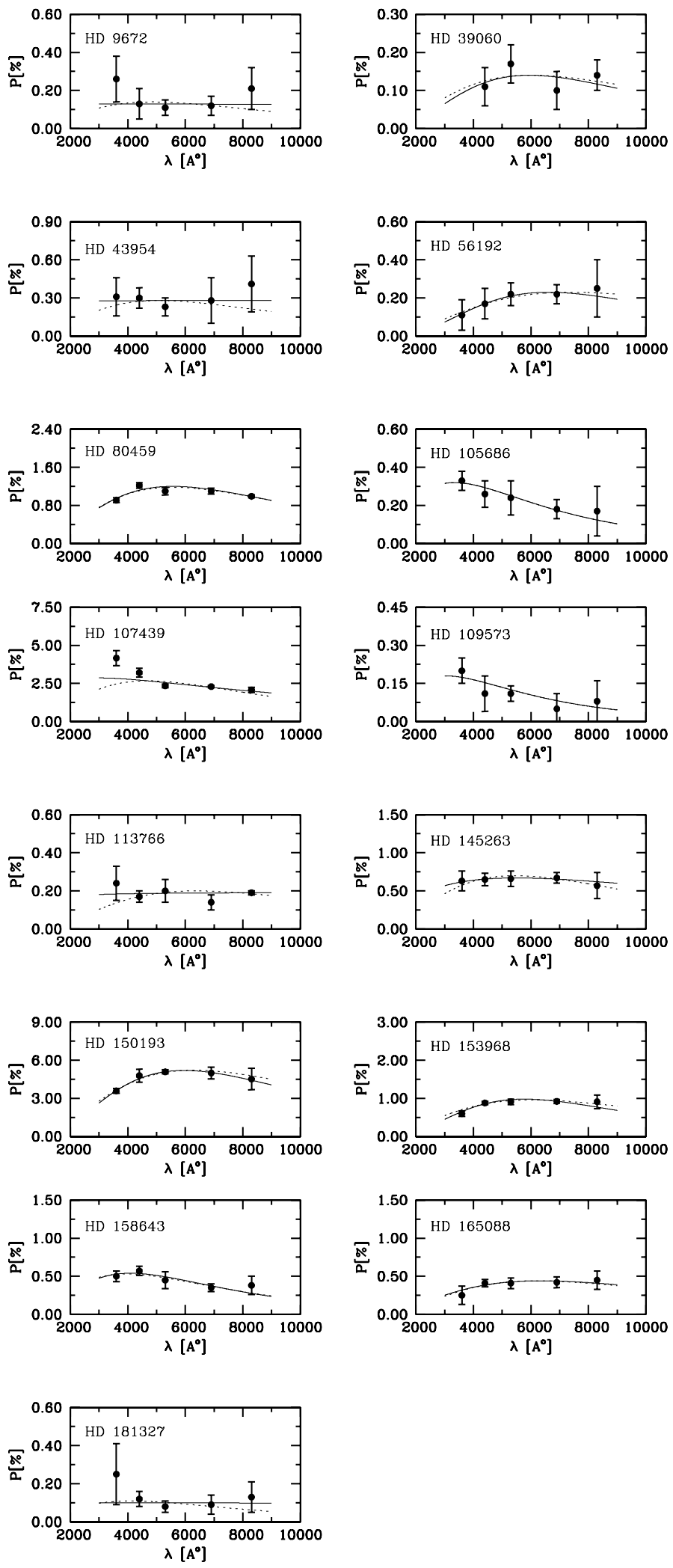

Fig. 5. Serkowski law fits for likely and marginally polarized sources (see Tables 2 and 3). The continuous line represents the three parameter model fitting ( $p_{\max }, K$ and $\lambda_{\max }$ ). The dotted line corresponds to a two parameter fit ( $\left.p_{\max }, \lambda_{\max }\right)$ to Eq. (1), adopting $K=1.15$.

analyzed the metallicity distribution of the complete sample in relation to the seven stars with far-infrared excess, finding no significant difference between the two groups.

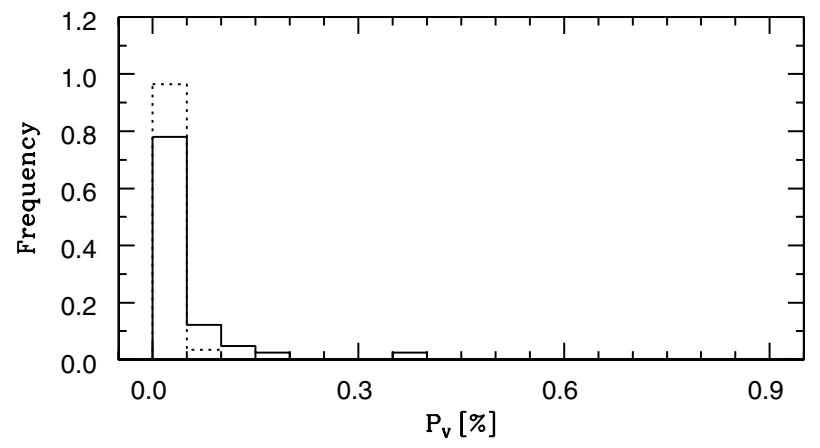

Fig. 6. Polarization distributions of IRAS infrared excess objects within $40 \mathrm{pc}$ of distance (continuous line) and for Exoplanet host stars (dotted line).

Our result may suggest that giant planets of the type detected by radial velocity techniques are not as common in IRAS infrared excess objects as in Exoplanet Host stars. Greaves et al. (2004) put forward an alternative suggestion based on the proposition that primordial disks associated with the EH stars were more massive than those in IRAS F and $G$ infrared excess objects. In disks with larger masses, the growing process of gas giant planets and inward migration is relatively fast in relation to the time scale for gas depletion. In contrast, disks with smaller masses evolve slowly. The new planet gains less mass and experiences outward migration that increases the collision rate of Kuiper Belt-like objects. Radial velocity techniques are not suitable to detect this type of large orbit and less massive planetary object. We caution, however, that the migration mechanisms is at the present time rather poorly understood. In addition, there is not obvious reason for massive disks not to form planets at large semi-major axis. These planets may also collide and stir up the Kuiper belt objects. In any event, small bodies at large distances would be harder to detect by the Doppler technique.

\section{Summary and conclusions}

We present new UBVRI polarimetry data for a group of 38 IRAS infrared excess objects and combine these observations with measurements for 87 additional stars from literature. After correcting the observed values by the interstellar contribution, we find that $48 \%$ of the analyzed sample has a polarization excess. The polarization of IRAS infrared excess stars may correlate with the infrared color excesses, particularly at 60 and $100 \mu \mathrm{m}$. We caution however that poor IRAS data quality affects fluxes at 60 and $100 \mu \mathrm{m}$. This fact casts some doubts on the reality of the detected correlation; better quality data (i.e., Spitzer Space Telescope observations) can help to clarify this issue.

We analyzed the wavelength dependence of the linear polarization of 15 observed objects that are likely or marginally polarized and find that 6 or 7 of these stars (depending on the model adopted for interstellar polarization) show polarization values that significantly differ from the empirical interstellar law, suggesting an intrinsic origin.

We constructed the polarimetry histogram distributions of IRAS infrared excess objects and Exoplanet host stars. The corresponding polarimetry distributions are different within a high confidence level. The group of IRAS infrared excess stars has a significant fraction of polarized objects (i.e., $41 \%$ of the sample) while all but one (Tau Boo, Heiles 2000) of the analyzed exoplanet host stars are consistent with non-polarized sources. 

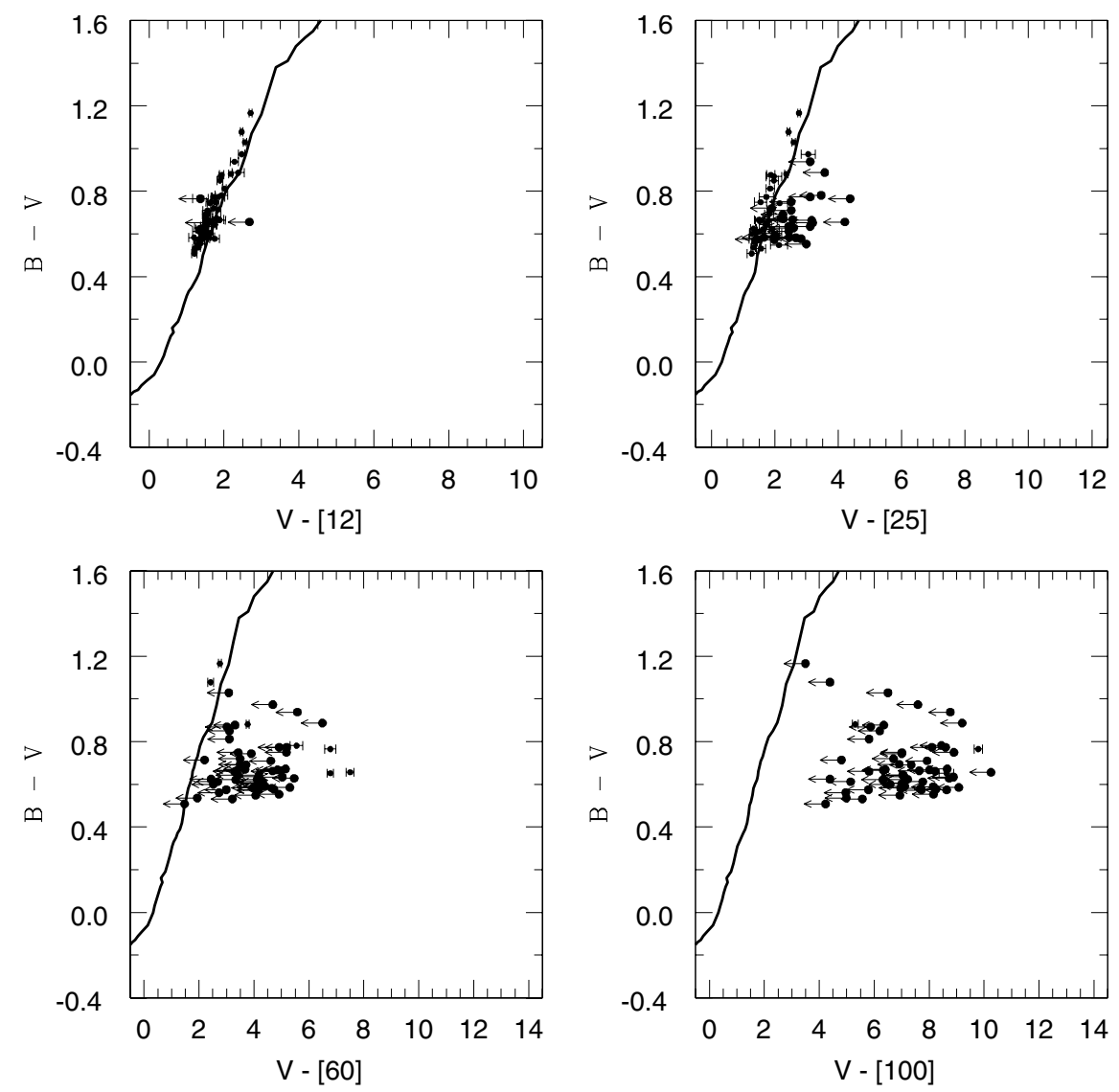

Fig. 7. $B-V$ vs. $V-$ [12], $V-$ [25], $V-[60]$, and $V-[100]$ color-color diagram for the Exoplanet host stars. The error bars correspond to the uncertainties in the IRAS fluxes. The arrows indicate objects for which only upper limits to the fluxes are given by the IRAS catalog. The solid line in the upper left panel indicates the location of main sequences stars (Kenyon \& Hartmann 1995). In the other panels we use a black body to calculate the main sequence. In these cases we adopted the calibration $T_{\text {eff }}$ vs. $B-V$ from the same authors.
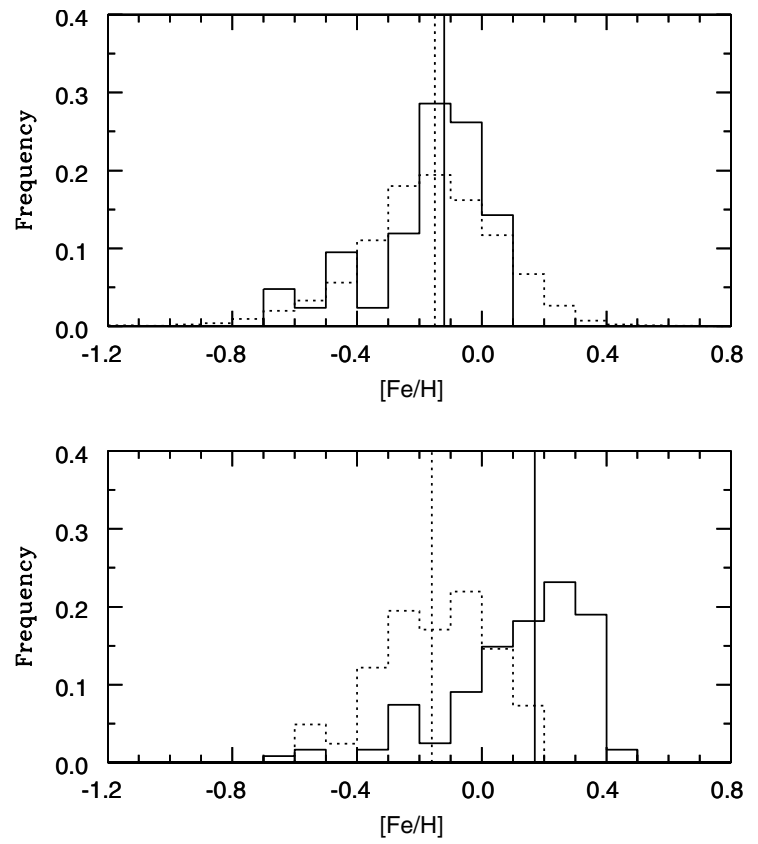

Fig. 8. Upper panel: Metallicity distribution of IRAS infrared excess objects (continuous line) and single field stars (dotted line), taken from Nordström et al. (2004). The median of each distribution $(-0.12$ and -0.15 , respectively) are indicated. In the lower panel we reproduce the metallicity distribution of Exoplanet host stars (continuous line) and main sequence stars not known to have planets (dotted line) from Santos et al. (2004). The medians, +0.17 and -0.16 respectively, are marked.
We compared the metallicity distributions of the three groups and found that IRAS infrared excess objects have metallicities quite similar (although not identical) to field main sequence stars and significantly different from the Exoplanet host stars. IRAS infrared excess objects and main sequence stars taken from the catalog of Nordström et al. (2004) have medians of -0.12 and -0.15 , respectively. Exoplanet host and field main sequence stars analyzed by Santos et al. (2004) have medians of +0.17 and -0.16 , respectively. IRAS infrared excess objects, on average, are not as metal rich as the Exoplanet host stars (see Santos et al. 2004, and the references therein). However, we caution that this conclusion is drawn from a sample of 42 IRAS infrared excess objects with spectral type $F$ and $G$ whereas most of this class of stars have A spectral type. On the other hand, lack of this spectral type makes the two analyzed samples more homogeneous.

Acknowledgements. This research has made use of the SIMBAD database, operated at CDS, Strasbourg, France. 2MASS and DENIS data were employed in this analysis. We are grateful to two anonymous referees for their objections, suggestions and comments that helped to improve this paper.

\section{References}

Aumann, H. H., et al. 1984, ApJ, 278, L23

Backman, D. E., \& Paresce, F. 1993, in Protostars and Planets III, ed. E. H. Levy, J. I. Lunine, \& M. S. Mathews (Tucson: Univ. Arizona Press), 1253

Bhatt, H. C., \& Manoj, P. 2000, A\&A, 362, 978

Beichman, C., Neugebauer, G., Habing, H. J., Clegg, P. E., \& Chester, T. J. 1988, IRAS Catalogs and Atlases Explanatory Supplement, (Washington, DC: GPO), NASA RP-1190, 1

Beichman, C. A., Bryden, G., Rieke, G. H., et al. 2005, ApJ, 622, 1160 
Berdyugin, A., Piirola, V., \& Teerikorpi, P. 2004, A\&A, 424, 873

Bryden, G., Beichman, C. A., Trilling, D., et al. 2006, ApJ, 636, 1098

Butler, R., Marcy, G. W., Vogt, S. S., et al. 2003, ApJ, 582, 455

Carpenter, J. M., Wolf, S., Schreyer, K., Launhardt, R., \& Henning, T. 2005, AJ, 129, 1049

Chen, C. H., Patten, B. M., Werner, M. W., et al. 2005, ApJ, 634, 1372

Dyck, H. M., \& Jones, T. J. 1978, AJ, 83, 594

Fajardo-Acosta, S. B., Stencel, R. E., Backman, D. E., \& Thakur, N. 1999, ApJ, 520,215

Fischer, D. A., \& Valenti, J. 2005, ApJ, 622, 1102

Fresneau, A. 1994, AJ, 108, 629

Gillett, F. C. 1986, in Light on dark matter; Proceedings of the First Infra-Red Astronomical Satellite Conference (Noordwijk, Netherlands: Dordrecht, D. Reidel Publishing Co.), 61

González, G. 1997, MNRAS, 285, 403.

González, G. 1998, A\&A, 334, 221

González, G., Law, C., Tyagi, S., \& Reddy, B. E. 2001, AJ, 121, 432

Gorlova, N., Padgett, D. L., Rieke, G. H., et al. 2004, ApJSS, 154, 448

Greaves, J. S., Holland, W. S., Moriarty-Schieven, G., et al. 1998, ApJ, 506, L133

Greaves, J. S., Holland, W. S., Jayawardhana, R., Wyatt, M. C., \& Dent, W. R. F. 2004, MNRAS, 348, 1097

Habing, H. J., Dominik, C., Jourdain de Muizon, M., et al. 2001, A\&A, 365, 545

Hauck, B., \& Mermilliod, M. 1998, A\&AS, 129, 431

Heiles, C. 2000, AJ, 119, 923

Helou, G. 1986, ApJ, 311, L33

Holland, W. S., Greaves, J. S., Zuckerman, B., Webb, R. A., \& McCarthy, C. 1998, Nature, 392, 788

Hsu, J.-C., \& Breger, M. 1982, AJ, 262, 732

Jayawardhana, R., Holland, W. S., Kalas, P., et al. 2002, ApJ, 570, L93

Judin, R. V. 2000, A\&AS, 144, 285

Kenyon, S. J., \& Hartmann, L. 1995, ApJS, 101, 117

Kim, S. H., Martin, P. G., \& Hendry, P. D. 1994, ApJ, 422,164

Korhonen, T., \& Reiz, A. 1986, A\&ASS, 64, 487

Laughlin, G., \& Adams, F. C. 1997, ApJ, 491, 51

Laureijs, R. J., Jourdain de Muizon, M., Leech, K., et al. 2002, A\&A, 387, 285

Leroy, J. L. 1993, A\&AS, 101, 551.

Lisse, C., Schultz, A., Fernández, Y., et al. 2002, ApJ, 570, L779

Mannings, V., \& Barlow, M. 1998, ApJ, 497, 330

Meyer, M. R., Hillenbrand, L. A., Backman, D. E., et al. 2004, ApJSS, 154, 422
Moshir, M., Copan, G., Conrow, T., et al. 1989, Explanatory Supplement to the IRAS Faint Source Survey, Version 2 (JPL D-10015 8/92; Pasadena: JPL) (FSC)

Murray, N., \& Chaboyer, B. 2002, ApJ, 566, 442

Nordström, B., Mayor, M., Andersen, J., et al. 2004, A\&A, 418, 989

Oudmaijer, R. D., Palacios, J., Eiroa, C., et al. 2001, A\&A, 379, 564

Perry, C. L., Johnston, L., \& Crawford, D. L. 1982, A\&AS, 87, 1751

Piirola, V. 1973, A\&A, 27, 383

Piirola, V. 1977, A\&ASS, 30, 213

Press, W. H., Teukolsky, S. A., Vetterling, W. T., \& Flannery, B. P. 1992, Numerical Recipes in Fortran: The Art of Scientific Computing, 2nd edition (Cambridge University Press), 617

Reid, I. N. 2002, PASP, 114, 306

Reiz, A., \& Franco, G. A. P. 1998, A\&AS, 130, 133

Sanders, D. B., Egami, E., Lipari, S., Mirabel, I. F., \& Soifer, B. T. 1995, AJ, 110,1993

Santos, N. C., Israelian, G., \& Mayor, M. 2001, A\&A, 373, 1019

Santos, N. C., Israelian, G., \& Mayor, M. 2004, A\&A, 415, 1153

Scaltriti, F., Cellino, A., Anderlucci, E., Corcione, L., \& Piirola, V. 1989, Soc. Astron. It. Mem., 60, 243

Schmidt, G. D., Elston, R., \& Lupie, O. L. 1992, AJ, 104, 1563

Schuster, W. J., \& Nissen, P. E. 2004, A\&A, 221, 65

Serkowski, K. 1963, ApJ, 138, 1035

Serkowski, K., Mathewson, D. S., \& Ford, V. L. 1975, ApJ, 196, 261

Sheret, I., Dent, W. R. F., \& Wyatt, M. C. 2004, MNRAS, 348, 1282

Simmons, J. F. L., \& Stewart, B. G. 1985, A\&A, 142, 100

Smith, B. A., \& Terrile, R. J. 1984, Science, 226, 1421

Soifer, B. T., Boehmer, L., Neugebauer, G., \& Sanders, D. B. 1989, AJ, 98, 766

Sylvester, R. J., Skinner, C. J., Barlow, M. J., \& Mannings, V. 1996, MNRAS, 279,915

Sylvester, R. J., \& Mannings, V. 2000, MNRAS, 313, 73

Tamburini, F., Ortolani, S., \& Bianchini, A. 2002, A\&A, 394, 675

Whittet, D. C. B., Martin, P. G., Hough, J. H., et al. 1992, ApJ, 386, 562

Whittet, D. C. B., Gerarkines, P. A., Hough, J. H., \& Shenoy, S. S. 2001, ApJ, 547,872

Wardle, J. F. C., \& Kronberg, P. P. 1974, ApJ, 194, 249

Wilking, B. A., Lebofsky, M. J., Kemp, J. C., Martin, P. G., \& Rieke, G. H. 1980, ApJ, 235, 905

Wilking, B. A., Lebofsky, M. J., \& Rieke, G. H. 1982, AJ, 87, 695 